\title{
EFECTOS DE LA ESTIMULACIÓN HIDRÁULICA (FRACKING) EN EL RECURSO HÍDRICO: IMPLICACIONES EN EL CONTEXTO COLOMBIANO
}

\author{
EFFECTS OF HYDRAULIC STIMULATION (FRACKING) ON WATER \\ RESOURCES: IMPLICATIONS IN THE COLOMBIAN CONTEXT
}

\author{
Sharel Charry-Ocampo* \\ Anibal J. Perez \\ Fecha de recepción: 23 de noviembre de 2016 \\ Fecha de revisión: 16 de abril de 2017 \\ Fecha de aprobación: 16 de junio de 2017
}

Cómo citar: S. Charry-Ocampo y A. J. Perez, "Efectos de la estimulación hidráulica (fracking) en el recurso hídrico: Implicaciones en el contexto colombiano," Ciencia e Ingeniería Neogranadina, vol. 28, no. 1, pp. 135-164. DOI: http://dx.doi.org/10.18359/rcin.2549

\section{RESUMEN}

La contribución de la extracción de gas lutita en la sostenibilidad energética ha sido evaluada de manera independiente, sin tener en cuenta su relación con la seguridad hídrica ni sus impactos en las fuentes de agua. Por esta razón, en la presente investigación se realizó un análisis de estos impactos a través de una revisión bibliográfica de carácter nacional e internacional. Si bien el análisis hecho sugiere dos aspectos fundamentalmente importantes: 1) impactos en el recurso hídrico asociados a la cantidad y calidad de este y 2) efectos sísmicos o sismicidad inducida, este estudio se enfoca principalmente en el primero. Los resultados de la revisión demuestran un creciente aumento de investigaciones sobre el impacto del fracking; sin embargo, se encontró que hay deficiencias en el uso de modelos numéricos

\footnotetext{
* Ingeniera Ambiental, Universidad Antonio Nariño. Asistente de investigación, Facultad de Ingeniería Ambiental, Universidad Antonio Nariño. Bogotá, Colombia. Correo electrónico: scharry@uan.edu.co. ORCID: https:// orcid.org/0000-0003-3068-9809

** Ingenero Civil y magíster en Hidrosistemas, Pontificia Universidad Javeriana, Bogotá, Colombia; doctor en Geociencias, Universidad de Tubinga, Alemania. Profesor e Investigador de la Facultad de Ingeniería Ambiental y Civil, y director del Grupo de Investigacion en Recursos, Ecologia, Desarrollo Sostenible e Ingenieria Ambiental (Gresia), Universidad Antonio Nariño, Bogota, Colombia. Correo electrónico: anibperez@uan.edu. co, anibaljoseperez@gmail.com. ORCID: https://orcid.org/0000-0001-5581-013X
} 
para cuantificar los efectos ambientales. Adicionalmente, los resultados muestran que los consumos asociados a la estimulación hidráulica no parecen ser significativos comparados con los usos típicos en otras prácticas económicas, como la agricultura (riegos). En este sentido, en el contexto colombiano los retos se basan en consolidar una mejor red de información piezométrica y en general un sistema de monitoreo más robusto para mejorar la toma de decisiones respecto al fracking, ya que este tipo de información representa el primer paso hacia la construcción de modelos hidrogeológicos que soporten la evaluación del riesgo y contribuyan a la reducción de la posible vulnerabilidad del recurso hídrico en el desarrollo de proyectos de aprovechamiento de gas lutita.

Palabras clave: fracking, recurso hídrico, efectos ambientales.

\section{ABSTRACT}

The contribution of shale gas extraction to energy sustainability has been evaluated independently regardless of its relation with water security and its impacts on water sources. For this reason, in the present investigation, we carried out an analysis of these impacts through a bibliographical review of national and international references. Although the analysis made suggests two fundamental aspects: 1) impacts on the water resource associated to the quantity and quality of this and 2) seismic effects or induced seismicity, this research focuses mainly on the first. The results of this review demonstrate a growing increase in research on the impact of fracking; however, it was found that there are deficiencies in the use of numerical models to quantify the environmental effects. Also, the results show that the consumption associated with hydraulic stimulation does not seem to be significant compared to typical uses in other economic practices such as agriculture (irrigation). In this context, in the Colombian context, the challenges are based on consolidating a better network of piezometric information and, in general, a more robust monitoring system to improve decision making regarding fracking, since this type of information represents the first step towards the construction of hydrogeological models that support the risk evaluation and contribute to the reduction of the vulnerability of the water resource in the development of projects of exploitation of shale gas.

Keywords: Fracking, water resources, environmental impacts.

\section{INTRODUCCIÓN}

El mejoramiento de los estándares de vida ha traído consigo el incremento de la demanda energética. Esta demanda ha sido atendida principalmente con energía proveniente de recursos no renovables como los hidrocarburos, los cuales han sido asociados comúnmente con perturbaciones ambientales que han derivado en contaminación de corrientes de agua superficial, acuíferos y costas. 
A mediados de los años 40, en Estados Unidos, se iniciaron las primeras alertas que Ilamaban la atención sobre el agotamiento de los recursos energéticos no renovables [1]. Esto generó una crisis de carácter energético que impulsó la exploración de otras alternativas para la obtención de hidrocarburos [2]. Como resultado de estas investigaciones, se descubrieron por primera vez hidrocarburos en yacimientos no convencionales (HYNC); rocas sedimentarias (en general lutitas) de naturaleza fracturada, donde se generaba gas natural (shale gas) [3].

Las lutitas son rocas sedimentarias de baja permeabilidad (nanodarcy), razón principal para que por muchos años fuera inviable la extracción y producción del gas almacenado [4]. Para enfrentar este problema se desarrolló la técnica de estimulación hidráulica (fracking), la cual permite generar permeabilidades adicionales en la roca, para así extraer el gas contenido en ella y la técnica de perforación direccionada que permite perforar pozos no verticales. Estas dos técnicas potenciaron la producción de gas en yacimientos no convencionales a escala industrial [5].

El aumento considerable de la explotación de gas de lutita ha generado una preocupación creciente en ambientalistas de todo el mundo, que ha derivado hasta en la prohibición de la práctica en algunos lugares como Francia o el Estado de Nueva York (Estados Unidos) y limitado con moratorias importantes en otros países como Escocia y Alemania [6]. Algunas de las razones expuestas por un amplio sector de ambientalistas están relacionadas con la contaminación de fuentes de agua debido a la inyección de sustancias tóxicas, explosiones durante el proceso de exploración, emisiones incontroladas de metano y hasta sismos localizados [7]-[9].

En este artículo se presenta una revisión bibliográfica de los efectos de la estimulación hidráulica en el ambiente, con especial énfasis en la literatura más actualizada, encaminada a describir el estado del conocimiento en esta temática. Dicha estimulación es de mucha relevancia en el contexto colombiano teniendo en cuenta que la estimulación hidráulica es un tema de controversia nacional desde 2014; primeramente, por los efectos sobre los recursos naturales, así como la tecnología por implementar, ya que actualmente seis departamentos cuentan con bloques cuyos contratos de exploración y producción hacen parte de los Proyectos de Interés Nacional Estratégico (PINE), siendo prioridad económica para el Estado [10].

En ese sentido, este trabajo representa una base importante hacia el reconocimiento de los principales riesgos ambientales de la explotación de gas en yacimientos no convencionales usando estimulación hidráulica, lo que puede aportar información guía para enfocar los esfuerzos investigativos de cara a los grandes retos ambientales a los que se enfrenta el país.

El presente artículo está estructurado de la siguiente manera: en la primera sección se exponen algunas generalidades relacionadas con el gas natural, las diferencias entre el gas natural convencional y el no convencional, la descripción detallada de cada etapa del proceso de estimulación hidráulica junto con los efectos ambientales 
asociados a estas prácticas. En este contexto se hace hincapié en la contaminación hídrica, sin dejar de lado la contaminación atmosférica, la contaminación del suelo y los efectos en la salud humana. En la segunda sección, se presenta una discusión de los efectos ambientales basados en datos expuestos por diferentes autores en la literatura, incluyendo el análisis de algunos esfuerzos en el uso de modelos numéricos aplicados a la explotación de gas de lutita, así como el planteamiento de algunas conclusiones y recomendaciones en el contexto colombiano.

\section{METODOLOGÍA}

La revisión sistemática del estado del arte de los efectos ambientales de la estimulación hidráulica es un trabajo que se ha venido desarrollando en diferentes, países con el fin de conocer las afectaciones del fracking en el medio ambiente. En este sentido, un esfuerzo importante fue realizado por Costa et al. [11], a través de una investigación del estado del arte mediante 328 artículos a nivel mundial que permitieron realizar una clasificación de ellos, de acuerdo con el impacto en seis categorías principalmente: 1) recursos hídricos; 2) emisiones atmosféricas; 3) uso del suelo; 4) sismicidad inducida; 5) salud ocupacional y pública y seguridad, y 6) otros impactos. Sin embargo, una de las principales conclu- siones de esta investigación fue la escasa cantidad de artículos referentes a la cuarta categoría (sismicidad inducida), puesto que en la ventana de tiempo 2010-2015, tan solo se encontraron ocho referencias que se pudieron analizar para demostrar un creciente aumento de las investigaciones sobre gas de lutita en Estados Unidos. De la misma manera, en el se consolidó aquí una metodología para una revisión sistemática del estado del arte de los efectos ambientales de la estimulación hidráulica que incluye las siguientes etapas.

\subsection{Búsqueda e identificación de artículos por palabras claves}

Para este fin se usó la palabra clave estimulación hidráulica (fracking). Esta búsqueda se hizo en la base de datos de Scopus, donde se encuentran relacionadas las referencias bibliográficas de mayor calidad. Los resultados arrojaron un total de 435 referencias, de las cuales más del $98 \%$ fueron publicadas después de 2009, como se puede observar en la Fig. 1.

Aproximadamente un $50 \%$ de las referencias analizadas corresponden a artículos de investigación, un $12 \%$ está relacionado con resúmenes de congresos o conferencias y un $10 \%$ con notas científicas. El $28 \%$ restante corresponde a las demás categorías, como se puede observar en la Fig. 2. 
Ciencia e Ingeniería Neogranadina

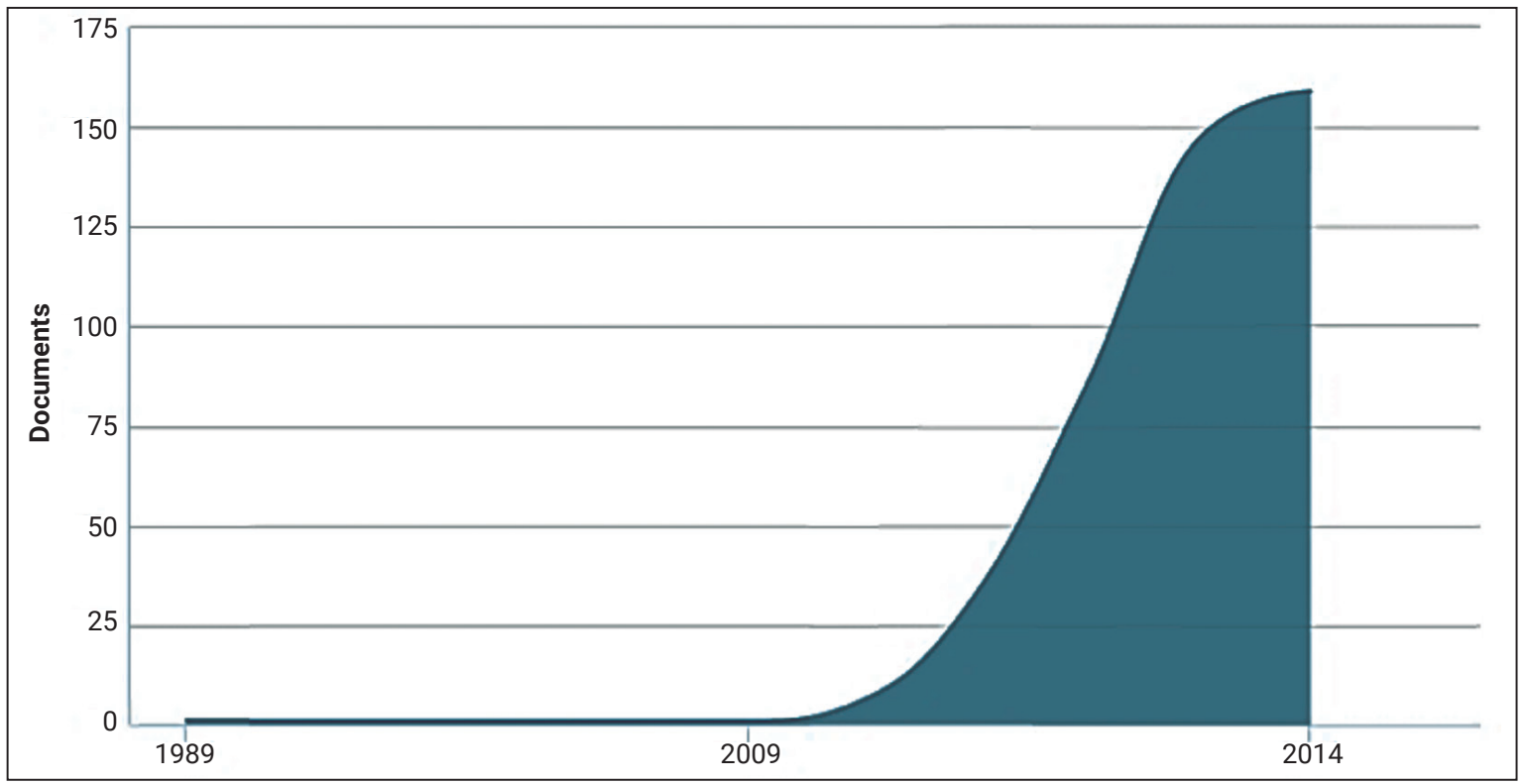

Fig. 1. Análisis de referencias por año de publicación

Fuente: elaboración propia.

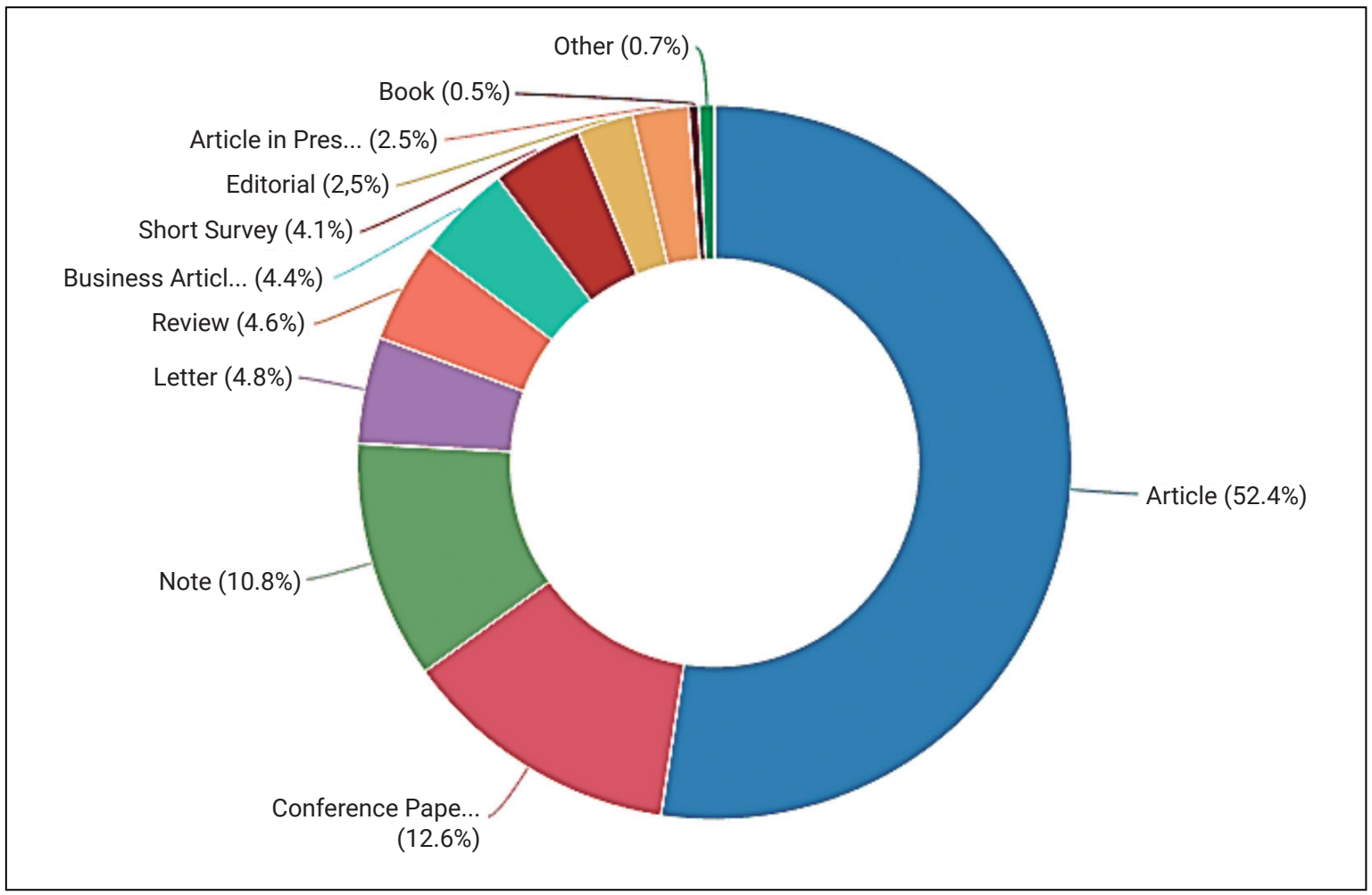

Fig. 2. Análisis de referencias por tipo de documentos

Fuente: elaboración propia. 
La mayoría de los documentos listados (133) ha sido publicadas en Estados Unidos, seguido de Alemania (33), el Reino Unido (29) y Canadá (11). En el análisis se puede observar que solo un documento ha sido publicado en Latinoamérica (en México), como se puede observar en la Fig. 3.
Al analizar la información por área de estudio, se observa que la mayoría de las referencias se concentran en ciencias ambientales (32\%), ingeniería (22\%), energía (19\%), ciencias sociales $(16 \%)$ y ciencias planetarias y de la tierra ( $15 \%)$, como se puede observar en la Fig. 4.

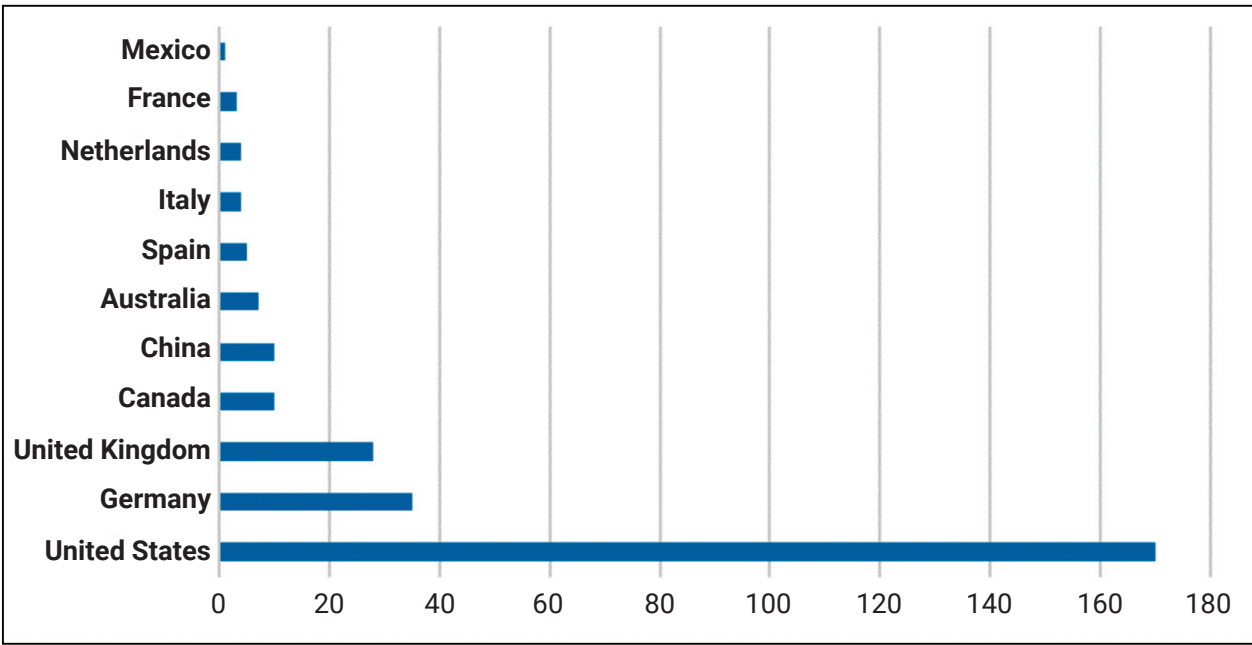

Fig. 3. Análisis de referencias por país de publicación

Fuente: elaboración propia.

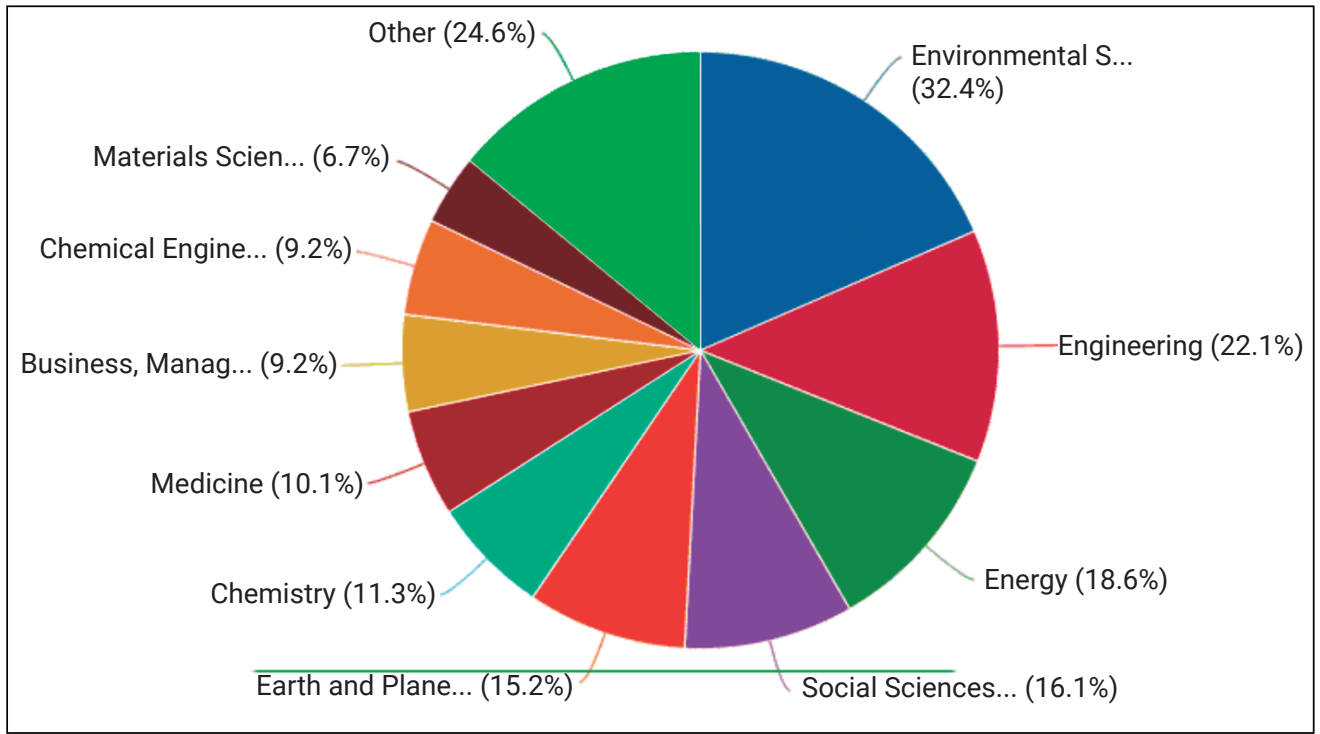

Fig. 4. Análisis de referencias por temática

Fuente: elaboración propia. 


\subsection{Clasificación de la información}

De las 435 referencias que componen el conjunto de referencias consultadas se preseleccionaron, después de leer los resúmenes de cada una de ellas, 150 que contenían algún tipo de información sobre efectos ambientales; estas 150 referencias se concentran en 45 revistas científicas. Dentro de este subgrupo, se seleccionaron 88 que contenían información relevante para efectos del presente artículo. Para complementar el análisis, se incluyeron en la revisión 6 referencias que, aunque no se encuentran en la base de datos de Scopus, brindan información que permite contextualizar la temática en el contexto regional y local.

\subsection{Análisis y catalogación del material bibliográfico}

Los 94 documentos seleccionados fueron catalogados en una matriz de información por orden cronológico y temático que incluye las diferentes categorías relacionadas con la estimulación hidráulica, las cuales son:1) efectos ambientales; 2) efectos en los recursos hídricos; 3) la estimulación hidráulica y la salud pública; 4) la estimulación hidráulica y el transporte de contaminantes, 5) simulación en la estimulación hidráulica, y 6) generalidades del shale gas. Esta matriz incluye un resumen de cada estudio y representa el punto de partida para la revisión de estado de arte presentada en el presente artículo.

\section{GENERALIDADES}

\subsection{Gas natural}

El gas natural es un término usado comúnmente para referirse a los gases provenien- tes de formaciones petrolíferas. Este gas es una mezcla de hidrocarburos livianos en estado gaseoso, que contiene en mayores proporciones metano, además de algunas parafinas $\left(\mathrm{C}_{n} \mathrm{H}_{2 n+2}\right)$ de peso molecular más alto en proporciones más pequeñas, como el etano, el propano, el butano y el pentano [12], [13]. También contiene componentes no inflamables como dióxido de carbono, nitrógeno y helio en trazas [12].

El gas natural es un recurso no renovable, el cual se quema para generar electricidad, y ofrece así una producción de alta eficiencia energética, de bajo costo y de combustión limpia en comparación con el carbón y el petróleo [14]. Es más seguro que la energía nuclear $y$, al contrario de la eólica y la solar, puede producir energía 24 horas, 7 días a la semana [7]. El gas fue encontrado por primera vez en 1821 en Fredonia, Estados Unidos; sin embargo, su uso fue restringido años después debido a la deficiencia tecnológica en los procesos de almacenamiento y transporte, así que su valor comercial bajó considerablemente. No es sino hasta la Segunda Guerra Mundial cuando el gas natural se convierte en un combustible popular [12].

El agotamiento del gas natural en los reservorios convencionales empezó a ser evidente a finales de los años 90, lo que exigió la identificación de otras fuentes de gas [3]. Así, los estudios promovidos por la escasez de gas natural convencional derivaron en el descubrimiento de la existencia de altos volúmenes de gas natural en la corteza terrestre [15]. Desde 2005, Estados Unidos comenzó el desarrollo de la extracción intensiva de gas natural no convencional conocido como 
shale gas o gas natural de lutita, lo que traería consigo beneficios importantes en su seguridad energética [16].

\subsection{Shale gas}

En general, las formaciones donde se ha encontrado gas en yacimientos no convencionales están asociadas a lutitas (shale) y a metano en capas de carbón (CBM) [17]. En la Fig. 5, se presenta un esquema de explotación de (a) gas en yacimientos convencionales y (b) shale gas. Como se puede observar, en los yacimientos convencionales la fuente, el depósito y la trampa se pueden diferenciar claramente, ya que son independientes. Por el contrario, en los depósitos no convencionales la fuente, el depósito y la trampa se encuentran combinados en una sola zona discreta. La fuente es una zona con rocas ricas en materia orgánica que, bajo condiciones de alta temperatura y presión, generan gas. El depósito es la roca donde se almacena el gas y tiene suficiente porosidad para trasmitir el fluido. La trampa es la roca impermeable que restringe el movimiento de hidrocarburos [18].

La forma como se encuentran en la naturaleza el gas convencional y el gas de lutita genera diferencias significativas en el proceso de explotación: en el caso de los yacimientos convencionales la explotación se hace directa, mientras que en de los no convencionales se requiere una perforación direccionada, además de una estimulación posperforación [18]. Estas actividades asociadas a la extracción del gas no convencional tienen implicaciones económicas importantes [16], [19].

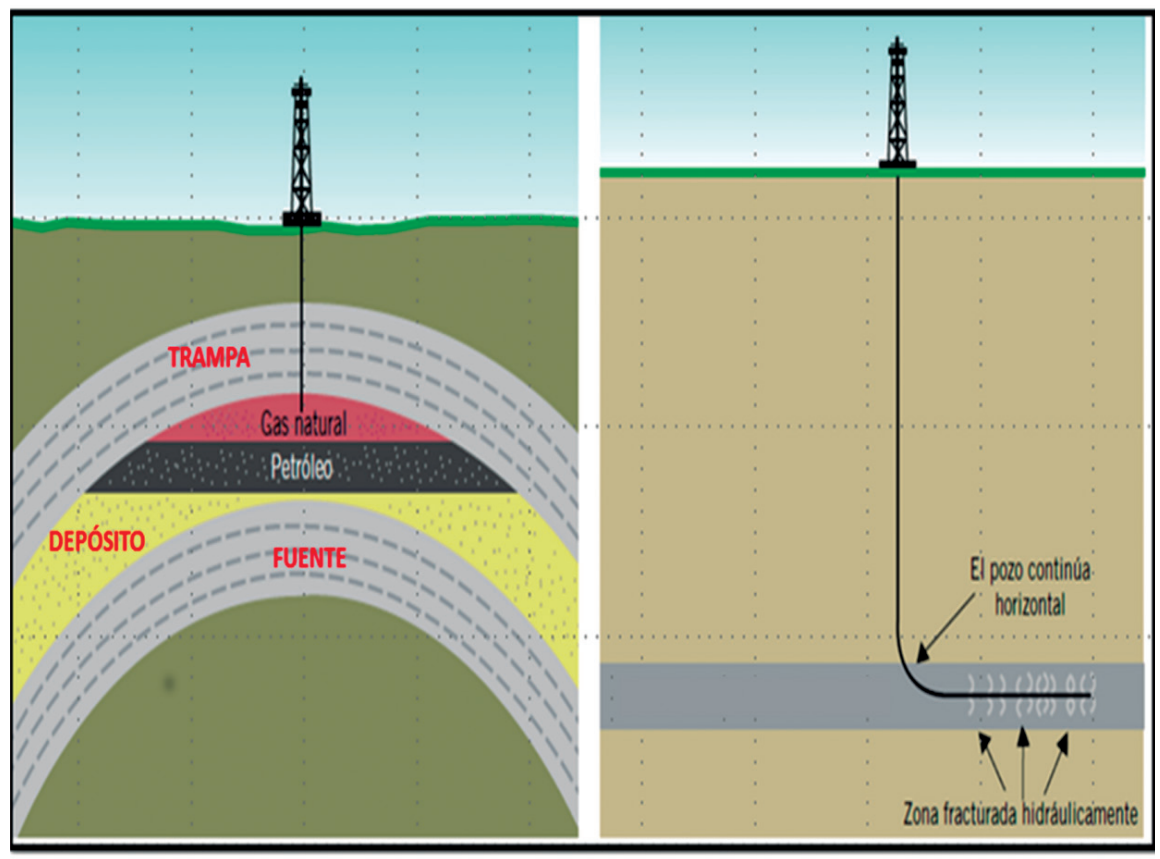

Fig. 5. Geología de yacimientos convencionales y no convencionales respectivamente: a) izquierda: gas convencional y b) derecha: gas de lutita

Fuente: [21]. 
Las shale son las rocas sedimentarias más abundantes en la corteza terrestre, que están compuestas de partículas de arcilla consolidadas [20], generadas como consecuencia de la deposición lenta y continua de sedimentos, principalmente marinos, que se encuentran a miles de metros debajo de la superficie de la tierra [14] Estas rocas contienen aproximadamente un $95 \%$ de materia orgánica; además, tienen la capacidad de absorber y liberar gran cantidad de humedad [21] que, bajo condiciones anóxicas, genera gas seco de tipo termogénico [20]. Este se acumula entre los espacios de los poros de la roca, dentro de las fracturas naturales o en la materia orgánica dentro del shale; es decir, se almacena en formaciones de muy baja permeabilidad [22].

Las características hidráulicas de una roca, como la permeabilidad y la porosidad, reflejan la habilidad para almacenar y transmitir fluidos líquidos y gaseosos [21]. Estas decrecen con la profundidad y compactación [23]. Los poros en estos yacimientos alcanzan típicamente $5 \times 105 \mathrm{~mm}$ de diámetro [24]. Asimismo, la permeabilidad está entre $10 \mathrm{y}$ 100 nd [23].

\subsection{Hitos históricos}

Durante el desarrollo de la estimulación hidráulica diferentes eventos determinaron su éxito industrial, entre los que se destacan los siguientes: en 1940, Floyd Farris de Stanolind Oil and Gas, estudió y diseñó una técnica de explotación de gas de lutita que incluía la adición de agua más un agente apuntalante que se introducía en la formación para ha- cer más eficaces los pozos de hidrocarburos [1]. La primera aplicación comercial se hizo en 1949 en un pozo cerca de Duncan (Oklahoma, Estados Unidos). Este experimento mostró resultados satisfactorios [2], [25], lo que hizo que la técnica se extendiera rápidamente y para 1970 se hizo popular en la industria petrolera.

Entre 1980 y 1990, George P. Mitchell, de Mitchell Energy \& Development, hizo otro aporte a la explotación en HYNC: descubrió la existencia de fracturas naturales en las lutitas. Con este conocimiento previo, en 1991 Mitchell fue el pionero en implementar perforaciones horizontales de más o menos un kilómetro de extensión [3]. En adelante, se desarrollaron y patentaron dos técnicas propiciadas por el progreso tecnológico: la perforación direccionada (vertical y horizontal) y la estimulación hidráulica (fracking) [16], los cuales se incorporan de manera combinada [7], [26].

En la misma década, se popularizó el uso de estas técnicas para la estimulación de la roca en el proceso de explotación de gas en yacimientos no convencionales [5]. De esta manera, se permitió aumentar la permeabilidad en la roca, lo que generó un incremento dramático en la extracción de gas no convencional a nivel mundial [4], [15], [27].

Existen más de 920 billones de metros cúbicos de gas natural no convencional en el mundo, casi el doble del gas natural hallado en yacimientos convencionales [7]. Según Groat y Grimshaw [16], en Estados Unidos la estimación actual de los recursos de shale gas es de 862 billones de metros cúbicos anuales, siendo el mayor y más importante productor de shale gas en el mundo. Energy 
Information Administration (EIA) [28] reportó la transición de la explotación de hidrocarburos convencionales a hidrocarburos no convencionales en Estados Unidos. Este estudio muestra cómo la explotación de shale gas ha aumentado desde 2009 hasta la actualidad y lo que aumentará en el futuro (30\% más hasta 2035).

Aunque China posee las más grandes reservas de gas no convencional en el mundo, apenas está alcanzado un adecuado nivel a escala industrial. Actualmente, se explota gas no convencional de la cuenca de Shanxi, Tarim, Songliao, Bohai Bay, Ordos, Junggar, Tuha, Qaidam, Qiangzhusi, longmaxi, siendo estas dos últimas las más abundantes [29]-[30].

\section{PROCESOS Y EFECTOS AMBIENTALES ASOCIADOS A LA EXPLOTACIÓN DE GAS DE LUTITA}

A continuación, se describen todos los pasos del proceso de explotación de gas de lutita, desde el momento cuando se dispone el área para explotar, hasta el desmonte de toda la operación.

\subsection{Exploración}

Es la etapa donde se determina el almacenamiento de gas en una formación [31] y la viabilidad técnico-económica que tendrá [32]. Se utilizan actualmente varias técnicas, pero las más comunes son los métodos sísmicos de tecnología geofísica [16], los cuales producen imágenes 3D del subsuelo y permiten guiar las perforaciones a los puntos adecuados de las formaciones [8], [16].

Primero, se perforan agujeros espaciados con profundidades de 5 a $20 \mathrm{~m}$, en los que se introducen cartuchos de dinamitas que explotan [32] y reflejan energía a los estratos del suelo. Esa energía se devuelve a la superficie y luego es medida por instrumentos llamados geófonos, que trasmiten la información a equipos de interpretación. Algunos autores recomiendan estudios fisicoquímicos adicionales de la formación [31]-[34].

Las explosiones sísmicas usadas típicamente durante las fases de exploración pueden producir fracturamiento en la roca y conectar acuíferos locales con acuíferos regionales que se encontraban previamente desconectados [35]. Asimismo, en esta etapa ocurre la emisión de sulfuro de hidrógeno (sustancia altamente tóxica [36]) como resultado de los estallidos, ya que este se encuentra naturalmente en la formación.

\subsection{Construcción}

Después de decidir la ubicación precisa, considerar algunas variables ambientales y sociales, y de tener los permisos necesarios, se realiza la instalación de los equipos. Primeramente, se estabiliza el área de los pozos, luego se construyen caminos de acceso en grava (entre 0,1 a 2,8 acres de longitud), se instalan las tuberías (conducción y producción) y las plataformas de perforación donde estarán los pozos [8], [21]. Finalmente, se ubican los contenedores o pozos superficiales, los lodos de perforación, los camiones y los materiales de perforación. 
Durante la fase de instalación, el tráfico de camiones se aumenta significativamente, por lo que se requieren entre 100 a 150 camiones con equipos de fracturación hidráulica y otros 100 a 1000 para cargar fluidos y algunos agentes químicos [37]. En la construcción se pueden producir efectos ambientales relacionados con la alteración del paisaje en áreas extensas. También, según varios autores, se pueden presentar cambios en la cobertura del suelo y transformaciones morfológicas que desequilibran la recarga de aguas subterráneas, cambios en los regímenes de flujo superficial, en los patrones de drenaje, en las tasas de escorrentía y en la calidad del recurso hídrico [1], [35], [38]-[41].

Durante la fase de construcción se ha documentado que se afectan también los ecosistemas naturales y consecuentemente especies típicas del lugar. Asimismo, se han encontrado amenazas a la biodiversidad biológica y cuando se involucra el recurso hídrico las poblaciones acuáticas [35], [40], [42]-[44]. En particular, la necesidad de despejar el área escogida para la extracción de gas afecta directamente la riqueza forestal, ya que la vegetación arbórea, arbustiva y herbácea es removida [16], [45]

Por otra parte, se han observado casos severos de erosión durante las fases de construcción de la infraestructura requerida para la explotación y por el aumento de tránsito vehicular [38], [42], [46]. El tráfico pesado también aumenta las concentraciones de gases en la atmosfera, lo que deteriora la calidad del aire local y contribuye al cambio climático [1], [27], [44], [46], [47].

\subsection{Perforación}

Los avances en el proceso de perforación fueron la clave para alcanzar un nivel de rentabilidad adecuada en la producción de gas de lutita [48]. La perforación se hace de manera direccionada, es decir, se empieza verticalmente y a determinada profundidad se desvía hasta alcanzar una posición horizontal en la formación que contiene el gas [8], [32] (ver Fig. 5).

La perforación consiste en atravesar las capas de la corteza terrestre utilizando barrenas o brocas de perforación hasta $15002000 \mathrm{~m}$ que es la profundidad a la que se encuentra el shale; típicamente esta distancia se encuentra por debajo de las fuentes potenciales de agua potable [24], [32], [48]. El pozo se va profundizando mediante rotación continua de la barrena (broca de perforación), que es apoyada de un fluido de perforación [49]. El fluido de perforación es utilizado para reducir la fricción, enfriar y lubricar la barrena y arrastrar hacia la superficie la roca triturada por la barrena [50]. Los fluidos de perforación pueden ser a base de agua o aceite y contener partículas sólidas como arcillas y coloides suspendidos en agua o salmuera para proporcionar viscosidad [49]-[51].

Durante la perforación se hace la instalación de tubos de revestimiento fabricados en acero y la cementación, cuya función es estructural [52], para así proteger el agua del subsuelo [16] y evitar que el pozo se derrumbe [32]. En general, se perforan varios pozos que son espaciados en intervalos de alrededor de 300 metros [24], los cuales son perforados consecutivamente y son distribuidos de tal manera que abarquen la totalidad del área determinada [52]. 
En la perforación se pueden presentar riesgos asociados a la calidad y alteración de las condiciones hidrológicas de los acuíferos de agua dulce, cuando se perfora a través de ellos [1], [53]. También pueden ocurrir vertimientos accidentales de contaminantes que pueden afectar la calidad del recurso hídrico [42], [46], [54].

Adicionalmente, la cementación defectuosa de la carcasa de revestimiento o problemas de integridad de esta pueden derivar en la migración de gas metano termogénico a los suministros de agua. Es posible que esta situación se presente durante todo el ciclo de vida del pozo y puede agravarse por las pulsaciones y vibraciones durante el proceso de perforación [8], [16], [35], [43], [55]. Por otra parte, las fugas de metano en la perforación pueden generar explosiones imprevistas en el subsuelo [16].

Existen otras emisiones de gases de efecto invernadero asociadas a la perforación, donde el gas principalmente emitido es el dióxido de carbono $\left(\mathrm{CO}_{2}\right)$ [46]. Además, los recortes de perforación son una fuente de salmuera y elementos radiactivos naturales que pueden comprometer la calidad de los recursos hídricos debido a su composición toxica [14], [42], [56], [57]. Por último, el cambio de presiones que se ocasiona durante la perforación puede producir sismos y hundimientos severos [8], [46].

\subsection{Fracturación (estimulación hidráulica)}

El propósito esencial de esta etapa es crear y mantener las fracturas en la roca para extraer el gas [3]. Después de que se ha perfo- rado el pozo se inserta en él un dispositivo que desata pequeñas cargas explosivas que generan grietas en la formación. Este dispositivo fue patentado en 1899 por el Coronel Edward Roberts [3], [58].

Seguidamente, se preparan los volúmenes del fluido de fracturación. Este tiene una composición que puede variar con cada explotación. Generalmente está compuesto por un $90 \%$ solución base, 9,5\% de agente apuntalante y $0,5 \%$ de aditivos químicos. La solución base puede ser agua, aceite o sintéticos. Los dos últimos fluidos son más estables y menos reactivos [14]; sin embargo los fluidos a base de agua son usados más comúnmente [8], ya que son asociados a menores impactos en el ambiente.

Aunque muchos aditivos químicos no son divulgados abiertamente por las compañías que practican esta técnica, los más comunes, según su función en el proceso, son: un biocida como el glutaraldehído [8], para evitar el crecimiento bacteriano que producen los gases; un inhibidor de incrustaciones como el glicoletileno, para evitar la precipitación del mineral [8]; un inhibidor de corrosión comola N-dimetilformamida [8], que impide la corrosión del tubo de acero, del revestimiento del pozo, de las herramientas y de los tanques [20]. Adicionalmente, se agregan sales como el cloruro de potasio entre $1 \%$ y $3 \%$ para controlar la inflación de la arcilla, ya que la permeabilidad se ve reducida significativamente, por lo que es expuesta a agua menos salina [22]. Igualmente, se adicionan geles orgánicos que apoyan al agente de apuntalamiento y aumentan la viscosidad del fluido [6], agentes tensioactivos como el metanol para aumentar la recupera- 
ción del fluido inyectado mediante la reducción de la tensión interfacial entre materiales, fluidos y formación [8], y eliminadores de oxígeno para evitar corrosión, como el ácido clorhídrico [22] o el bisulfato de amonio [8].

El agente apuntalante es un material granular que generalmente es arena, se utiliza para sujetar o mantener abierta la fractura y permitir que el gas fluya. Otros agentes de sostén de uso general son arena recubierta de resina, cerámica, bauxita u óxido circonio sintetizado [20], [22].

Después de tener listo el fluido de fracturamiento, se dispone de un equipo especializado que está equipado con tanques de almacenamiento, bombas, camiones químicos, tuberías y accesorios para facilitar la conexión de todos los elementos [48], [58]. Allí el fluido de fracturación es bombeado a alta presión (10000 a 20000 psi [59]) a través de la perforación, con el fin de producir y ampliar las finas fracturas en el sustrato rocoso, de tal manera que se aumente la superficie de contacto de los poros que encierra el gas [3], [6], [24].

Luego de la apertura de las fracturas en la formación, se reduce la presión, lo que crea un retorno del fluido (fluido de retorno) que luego se pasa por una purga donde se separan los distintos componentes. Finalmente, se aplica un ácido que sirve para limpiar la zona cercana al pozo, después del proceso de fracturación [22]. En los últimos años se ha venido desarrollando la fracturación de alto volumen (High Volume Fracturing HVF ), en la cual cada pozo se puede fracturar de 15 a 20 veces consecutivas, y desde una sola plataforma pueden derivar hasta 6 pozos independientes [14], [58], [59].
Los volúmenes de agua necesarios para generar las fracturas de manera artificial son típicamente provistos por caudales de arroyos, lagos, ríos y fuentes subterráneas [39]. Muchos autores [1], [40], [43], [53], [60] han reportado que esto ha traído como consecuencia la reducción del caudal estacional, la degradación en la calidad del agua regional y la reducción del nivel freático; además, ha generado conflictos ligados a la competencia del recurso con la agricultura, ganadería y suministro de agua potable [38], [39], [53].

Otra preocupación de la etapa de fracturación surge de la posibilidad de que una fractura hidráulica se conecte con la superficie, debido a la propagación de fracturas inducidas por fuera de la zona de las lutitas [16], [61]. Por ejemplo, puede ocurrir que una fractura hidráulica intercepte una fractura natural o una falla geológica, y crear así vías hidrodinámicas conductoras de los compuestos del fluido usado para estimulación hidráulica acuíferos poco profundos que conectan a pozos o redes domésticas [5], [43], [62]-[64]. Existe el riesgo, además, de que al fracturar el lutita se facilite la migración de sustancias tóxicas presentes en el subsuelo, como mercurio, radio, plomo y arsénico [38], [46].

El ingreso del fluido de fracturación a las formaciones puede aumentar la ocurrencia de reacciones químicas y la creación de nuevas sustancias que también llegarían potencialmente al recurso hídrico [46].

\subsection{Producción y distribución}

En esta etapa, el gas producido es separado y tratado preliminarmente para luego ser distribuido por gasoductos [32]. Aquí se debe 
considerar el manejo de tres fluidos distintos: primero, las cantidades de agua dulce para la estimulación hidráulica, que normalmente se dispone en pozos evitando la filtración al suelo; en segundo, lugar está el almacenamiento y la gestión de los aditivos químicos; y en el último lugar está el manejo del fluido de retorno [48].

El fluido de retorno generado después de la estimulación hidráulica es llevado a la superficie; este puede ser entre el $20 \%$ y el $80 \%$ del fluido usado para el fracturamiento [16]. Este fluido se almacena temporalmente en embalses o camiones para ser reciclado, reutilizado, tratado o para su eliminación [16]. Igualmente se gestionan y manejan los residuos sólidos y las emisiones de gases de efecto invernadero [16]. La contaminación hídrica asociada a este proceso puede responder a varias razones: 1) prácticas incorrectas; 2) daños en la estructura del pozo, y 3$)$ derrames accidentales y fugas [41], [46], [62], [65], [66].

El fluido de retorno puede llegar por derrames a aguas superficiales o mediante fugas migrar a acuíferos poco profundos. Además de los aditivos químicos ya contenidos en el fluido de fracturación, el fluido de retorno contiene altos niveles de salinidad, materiales radiactivos naturales (NORM), así como metales pesados, compuestos orgánicos volátiles, entre otros [9], [45], [62], [67]-[71]. La inadecuada gestión del fluido de retorno puede ocasionar contaminación en el suelo, deteriorar la calidad del recurso hídrico y producir efectos crónicos sobre los organismos acuáticos [46].

Los impactos sobre la salud humana se derivan de la ingestión de agua contaminada, de procesos respiratorios o por contacto cutáneo [69], cuyos casos más graves están asociados con el desarrollo de cánceres [27], [35], [42], [67], [72].

Durante la vida útil de los pozos existen emisiones fugitivas de gas metano en la perforación, el transporte, el almacenamiento, desde los fluidos de retorno y en la distribución a través de las tuberías urbanas, además de la emisión de otros gases. Esto influye directamente en la calidad del aire local [53], [55], [66], [73].

La fauna también ha sufrido las consecuencias de la estimulación hidráulica evidenciadas con la alteración de su ciclo reproductivo, pérdida de pelaje y algunas enfermedades [67], [74], [75].

\subsection{Recubrimiento y finalización del pozo}

La vida productiva típica de un pozo se acerca a los 40 años [76]. Después de que la producción de gas en los pozos se agota, estos son cementados en el fondo, luego se desinstalan los equipos [32], es decir, se desmantela y sella [52], para luego restaurar las áreas.

Algunos de los efectos ligados a esta etapa del proceso son los altos niveles de acidez y salinidad que quedan en el suelo, lo que inhibe el crecimiento de las plantas [38].

Por último, los sistemas de producción de gas de lutita requieren de 3 a 6 años para llegar a un nuevo equilibrio; esto implica que los cambios significativos causados por la estimulación hidráulica transporten advectivo de contaminantes en los acuíferos por 
lo menos por diez años más después de la finalización del pozo [24].

\section{DISCUSIÓN}

\subsection{Consumo hídrico}

Uno de los aspectos que genera más controversia en lo relacionado con la estimulación hidráulica tiene que ver con los recursos hídricos, principalmente con el consumo de elevadas cantidades de agua [9]. Los valores de consumo reportados en la literatura presentan una gran variación, como se puede observar en la Tabla 1, oscilando entre 3785 y $75708 \mathrm{~m}^{3}$ por pozo [77]. Las variaciones pueden ser explicadas por las diferencias en la profundidad y la distancia horizontal por recorrer, es decir, por las características propias de cada pozo [66].

Según el Servicio Geológico de Estados Unidos. se consumen aproximadamente $1,30 \times 10^{6}$ millones $\mathrm{m}^{3}$ anuales de agua dulce en este país, de los cuales los mayores porcentajes de consumo corresponden a la industria termoeléctrica y al riego [78]. En este orden de ideas, el consumo de una explotación como Marcellus de \pm 25 millones de metros cúbicos/año y el de Barnnet con

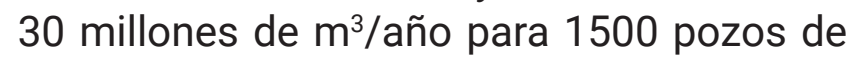
extracción no parecería ser significativo; sin embargo, el crecimiento desmedido de pozos puede poner en riesgo la sostenibilidad del recurso hídrico [43], [79]. Esto es particularmente grave en regiones donde la oferta hídrica es limitada y donde existe competencia con el suministro de agua potable.

Tabla 1. Consumo de agua para desarrollar fracking

\begin{tabular}{|l|l|l|}
\hline \multicolumn{1}{|c|}{ Autor } & \multicolumn{1}{|c|}{ Año } & \multicolumn{1}{c|}{ Consumo recurso hídrico/pozo $\left(\mathbf{m}^{3}\right)$} \\
\hline Jackson & 2014 & $7570 a 75708$ \\
\hline Brantley et al. & 2014 & 15142 a 18927 \\
\hline Vidic et al. & 2013 & 7570 a 26498 \\
\hline Peduzzi \& Harding & 2013 & 7570 a 30283 \\
\hline Eaton & 2013 & 7570 a 37854 \\
\hline Rivard et al. & 2013 & 7500 a 15000 \\
\hline Freeman & 2013 & 22712 \\
\hline Myres & 2012 & 17034 \\
\hline Groat y Grimshaw & 2012 & 15142 a 23091 \\
\hline Bocora & 2012 & 18927 \\
\hline Weijermars et al. & 2011 & 4542.5 a 13249 \\
\hline D. Rahm y Jiang et al. & $2011-2012$ & 7570.8 a 18927 \\
\hline Harper & 2008 & 15141.6 \\
\hline
\end{tabular}

Fuente: elaboración propia. 


\subsection{Fluido de fracturamiento}

Respecto al fluido de fracturamiento en Estados Unidos, entre 2005 y 2009 , las industrias de la estimulación hidráulica usaron 94 millones de galones de aditivos químicos que contienen 279 distintos compuestos [65]. Asimismo, la Comisión Europea clasificó 270 sustancias usadas en la estimulación hidráulica, de las cuales 58 son "preocupantes para la salud humana y para el medio ambiente" [40]. Entre 1982 y 2011, en Alemania, se analizaron 80 productos usados en estimulación hidráulica, de los cuales 6 resultaron ser tóxicos, otros 6 peligrosos para el medio ambiente, 25 nocivos para la salud, 14 irritantes y 12 corrosivos. Los 27 restantes no son considerados como peligrosos. Otro estudio [6] categorizó tres productos de los usados en el fluido de fracturamiento como extremadamente peligrosos para las aguas subterráneas.

\subsection{Contaminación hídrica superficial y subterránea}

Tanto los fluidos de fracturamiento como los fluidos de retorno representan un riesgo potencial para las aguas subterráneas y superficiales, debido a la conexión existente con zonas de recarga locales y regionales [54]. Sin embargo, las investigaciones realizadas no han determinado con exactitud las fuentes de contaminación [7], [24], [46].

El American Petroleum Institute (API) en 1980 desarrolló un método para calcular el riesgo de que los fluidos de fracturamiento pudieran alcanzar una fuente potable. La probabilidad arrojó un valor muy bajo $\left(2 \times 10^{-5}\right)$ [22]. No obstante, investigaciones posterio- res sugieren que esta probabilidad fue subestimada: desde 1982 hasta 2013 se han reportado 100 pozos subterráneos contaminados [43] y dos acuíferos (Catskill y Lock Haven), localizados en el noreste de Pennsylvania, sugieren una posible migración de salmueras de la formación Marcellus a través de vías naturales [54]. Adicionalmente, la literatura hace referencia a más de $30.2832 \mathrm{~m} 3$ de fluidos de fracturamiento vertidos al subsuelo en los Estados de Pennsylvania, Wyoming y Ohio [66]. En los mismos Estados se han reportado problemas con acumulación de gas en pozos domésticos de agua dulce [43], explosiones [16] y vertidos intencionales de fluido de retorno a humedales y a ríos [45], [46].

Se han reportado cambios isotópicos en algunos pozos domésticos y aguas subterráneas, que presentan elevados valores de salmueras [54], [71]. También se han encontrado compuestos sintéticos orgánicos, cloruro, potasio y acrilonitrilo en niveles que superan entre 13 y 1300 veces el nivel máximo permitido para agua potable [42], [46], [53], [56]. Dos estudios [24], [62] reportaron altas concentraciones de metano cerca de los lugares de explotación de gas lutita en el agua de pozos subterráneos y en el aire de algunos sótanos. Del mismo modo, en el Condado de Jackson en Virginia Occidental, monitoreos en el Rio Monongahela mostraron una elevada concentración de sólidos disueltos totales [8], [45].

Para disminuir el impacto de la contaminación de fuentes hídricas, las industrias han hecho esfuerzos importantes que incluyen tratamientos como destilación, evaporación y cristalización, separación por hidrociclones, electrocoagulación, resinas 
de absorción y ósmosis inversa. Esta última práctica puede mejorar la calidad del fluido de retorno hasta en un $80 \%$, pero los costos asociados son muy elevados [40], [67], [80].

Jackson et al. [59] reportaron que anualmente se producen casi $3,8 \times 109 \mathrm{~m} 3$ de aguas residuales generadas por actividades vinculadas a petróleo, gas de yacimientos convencionales y no convencionales.

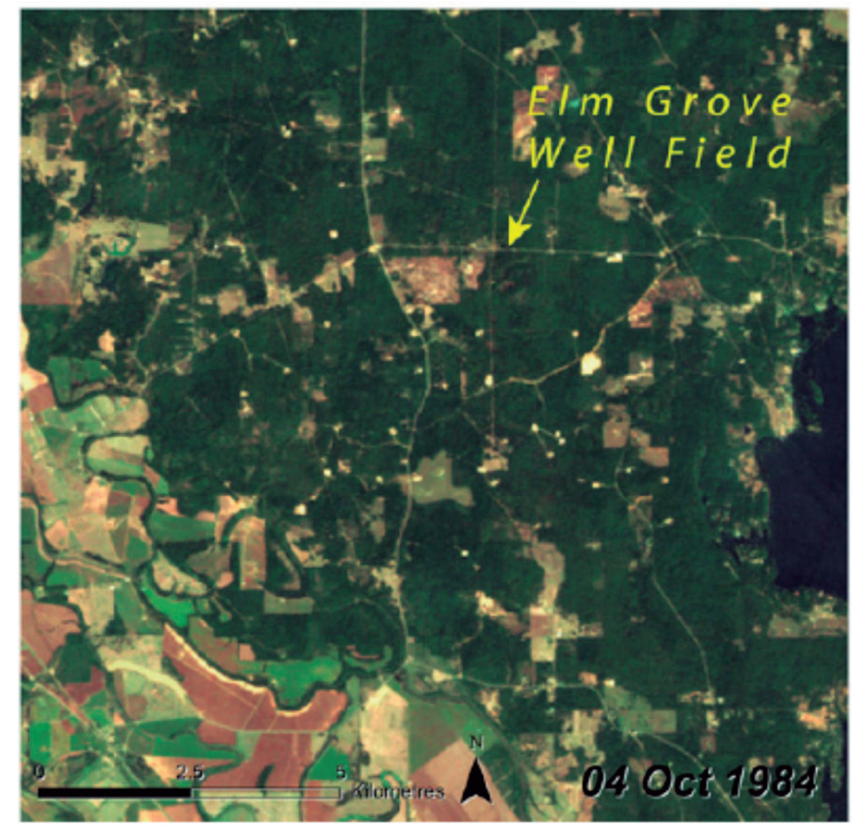

a) 1984

\subsection{Efectos en el suelo y aire}

La producción de gas de lutita afecta a los bosques a gran escala. Por ejemplo, en Marcellus Shale, 2/3 de las plataformas fueron construidas en claros de bosque, lo que obligó a talar de 34.000 a 83.000 ha [16]. Este ejercicio derivó en un impacto importante en los patrones de uso del suelo [38] (Fig. 6).

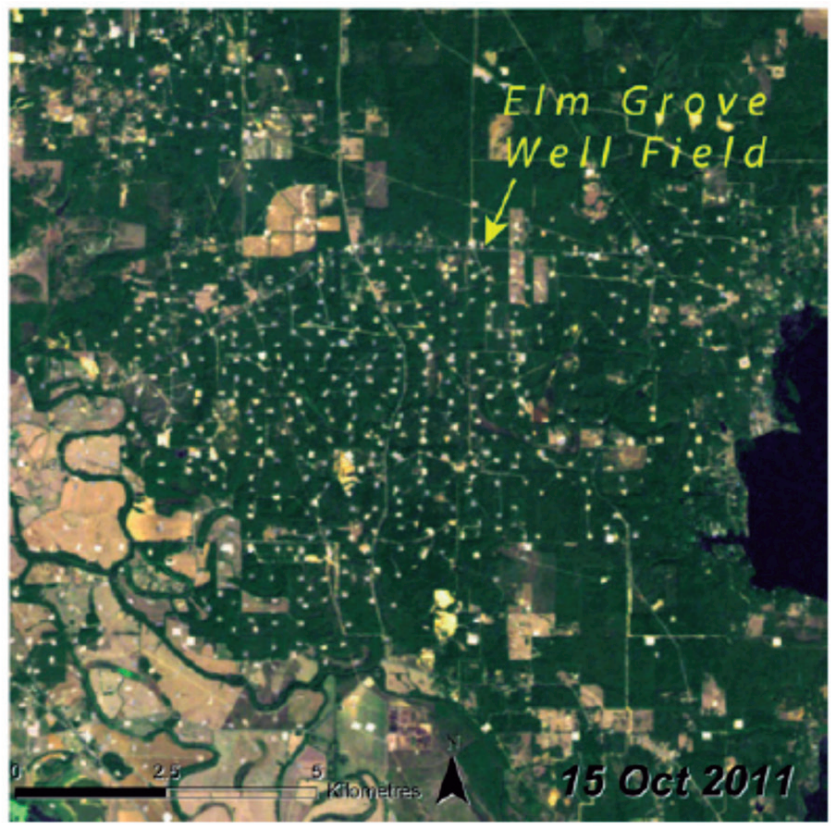

b) 2011

Fig. 6. Transición del cambio del suelo en una zona de Luisiana en Estados Unidos Fuente: [38].

Existen emisiones de gases durante todas las etapas de la explotación del gas de lutita. Clarkson [81] mencionó que el gas de lutita, comparado con otros combustibles fósiles, no mitiga el efecto del calentamiento global. En particular, la producción de gas no convencional emite entre $40 \%$ y $60 \%$ más gases de efecto invernadero (GEI) que la producción de gas convencional [7]. También se ha reportado que en un periodo de 20 años el gas de lutita tendrá una mayor huella de GEl que el carbón [46], [55]. De la misma manera, Bunch et al. [72] sugirieron que las mayores emisiones antropogénicas de metano en Estados unidos provienen de la extracción y transporte de gas, lo que afecta la calidad del aire local. 


\subsection{Sismicidad inducida}

De acuerdo con algunos autores [38], [63], [67], la sismicidad local se ha visto afectada por los cambios de presiones o tensiones en la corteza terrestre, que derivaron de actividades relacionadas con la estimulación hidráulica. En particular, en Cleburne (Texas) durante el 2009 se registraron siete sismos entre junio y julio [63]; en Blackpool (Reino Unido) se presentó un sismo de 1,5 grados en la escala de Richter [46]; en Oklahoma (Estados Unidos) se han reportado 50 sismos de pequeña magnitud [38]. Estos movimientos pueden acelerar el transporte de contaminantes por medio de las fallas geológicas, lo que puede derivar en el deterioro de la calidad de recursos hídricos subterráneos.

El Servicio Geológico de Estados Unidos (USGS) [82] publicó un estudio acerca de sismicidad local inducida por actividades humanas, en el cual se evidenció que los sismos han aumentado su ocurrencia desde el año 2000 hasta 2009. Algunos científicos del USGS han elaborado modelos preliminares para la predicción de la probabilidad de sismos en un tiempo de simulación de 50 años, que sirven como soporte para la toma de decisiones en algunos estados. La mayoría de estos sismos se encuentran en el rango de magnitud 3-4 en la escala de Richter; sin embargo, en Trinidad (Colorado), se presentó un sismo de 5,3, atribuido a la reinyección de aguas residuales producto del fracking correspondiente a fluido de fracturación mezclado con salmueras propias de la formación; el más alto asociado al fracking que se haya reportado. De la misma manera, existen otros sismos representativos, pero que aún no se atribuyen oficialmente a una causa inducida; por ejemplo, en Oklahoma se presentó un sismo de 5,6 en la escala de Richter en 2016 y de acuerdo con una serie de estudios en Estados Unidos, se presume que este evento fue inducido dada la cantidad de pozos de inyección cercanos al área. Un estudio muy importante en este contexto, en el que se analiza la sismicidad inducida a través del análisis de los pozos de inyección cercanos a los lugares de ocurrencia de los sismos, fue desarrollado por la USGS e incluye un modelo para la clasificación de los sismos entre naturales e inducidos. Asimismo, en este estudio se analizan las variables disponibles de ocurrencia de un fenómeno sísmico, como son la duración, el periodo de recurrencia y la magnitud [83].

Otros autores [84], [85] también asocian los eventos sísmicos a la reinyección de aguas residuales, más que por la fractura de la lutita.

\subsection{Modelación numérica}

Durante el desarrollo de las actividades de exploración y explotación de gas de lutita se han realizado algunos estudios que usan modelos conceptuales y numéricos en diferentes perspectivas. Sin embargo, la mayoría de los esfuerzos en modelación numérica en el contexto de la explotación de gas de lutita han sido enfocados en la optimización de las fases de producción. Estos estudios incluyen datos como madurez térmica, contenido orgánico, comportamiento del flujo darciano de agua y gas en los reservorios, cambios de presiones, litología, saturación de fluidos, regímenes de tensión, entre otros [20], [76], [86], [87]. 
Myres [24] realizó un análisis y una caracterización de los factores asociados al transporte de contaminantes en las lutitas hacia los acuíferos cercanos a través de vías naturales, considerando los tiempos de desplazamiento de los contaminantes, las vías posibles de transporte y parámetros hidrogeológicos locales. Como conclusión de este estudio, las simulaciones sugieren que el tiempo de transporte de contaminantes podría verse disminuido en las escalas de tiempo geológico, así como el flujo preferencial a través de las fracturas naturales también podría disminuir aún más los tiempos de transporte de contaminantes por pocos años.

Otras investigaciones [5], [88]-[90] que cuantifican los impactos de la estimulación hidráulica usando modelos numéricos fallan en la construcción de un modelo integrado que permita analizar los impactos de los procesos de exploración y explotación de gas de lutita. Este tipo de enfoques integrales representa uno de los grandes retos ambientales en los países donde se viene explotando este recurso y uno aún mayor en los países que apenas inician estos procesos.

\subsection{Contexto nacional}

La Agencia Nacional de Hidrocarburos (ANH) y el Congreso para el Desarrollo de los No Convencionales, Shale Colombia 2013, publicaron que las cuencas colombianas contienen grandes volúmenes de reservas aprovechables de gas en yacimientos no convencionales. En la zona de Catatumbo y del Cesar existen aproximadamente 31,7 trillones de pies cúbicos (TFC) y en la zona del Valle Medio del Magdalena alrededor de (VMM) 29 TFC.

La implementación de estas nuevas técnicas de extracción de hidrocarburos en yacimientos no convencionales aún es un desafío para el país. Las entidades de orden nacional y regional que regularan esta práctica deben considerar que los estudios y políticas que hay respecto al fracking están pensados para otros países cuyas condiciones hídricas y geológicas no son similares a las colombianas. Por ejemplo, en Estados Unidos se regulan los riesgos del fracking sobre el recurso hídrico subterráneo, con planes de manejo ambiental de agua subterránea en acuíferos de baja profundidad. En Colombia las condiciones serían distintas porque ya se está extrayendo agua en acuíferos de más de 1000 $\mathrm{m}$ [91]; es decir, si las perforaciones verticales típicas de la estimulación hidráulica $\geq 1000$ m [92], [93] se ejecutan, claramente se comprometería el estado de los recursos hídricos subterráneos con mayor probabilidad.

Para la protección de los recursos naturales, y los posibles riesgos por actividades de fracking, se deben considerar las siguientes políticas nacionales:

- El Código Nacional de Recursos Naturales Renovables y Protección del Medio Ambiente, en el artículo 52, decreta que cuando se compruebe que las aguas del subsuelo estén en peligro de agotamiento o de contaminación se suspenderán las actividades asociadas.

- La Resolución 90341, en el artículo 16, decreta la suspensión de actividades de inyección de agua residual proveniente de explotación de hidrocarburos 
en yacimientos no convencionales, en caso de eventos sísmicos mayores o iguales a 4 grados en la escala Richter, cuyo epicentro esté ubicado en el área de extracción.

- El principio de precaución en la actividad relacionada con el fracking cuando existe un riesgo latente para el patrimonio ambiental del país (Contraloría General de la Republica).

En particular, la ejecución de este tipo de extracción trae varios riesgos sísmicos para Colombia, ya que tiene una geología constituida por varios sistemas de fallas donde particularmente las mayores reservas de esta clase de recursos están en zonas de alta y media sismicidad [94]. Si se estimulan estas fallas es probable que el aumento de la actividad sísmica sea aún más recurrente y perjudicial para Colombia que en el caso de Estados Unidos. Noguera [95] recientemente notificó 976 sismos de pequeña escala ocurridos entre 2013 y 2016 en Puerto Gaitan (Meta), generados probablemente por el aumento de la actividad petrolera debido a la reinyección de aguas residuales. Este reporte en particular aumenta las preocupaciones respecto a las futuras reinyecciones de aguas residuales generadas por fracking.

En Colombia, como a nivel mundial, es notable que el desarrollo de la estimulación hidráulica está influenciado principalmente por factores económicos y políticos. Actualmente seis departamentos cuentan con bloques cuyos contratos de exploración y producción hacen parte de los PINE, siendo prioridad económica para el Estado, recursos que a partir del Sistema General de
Regalías (SGR) serán destinados a inversión social y posconflicto [10].

\section{CONCLUSIONES}

Las investigaciones geológicas en el mundo sobre las verdaderas reservas de HYNC aún se desconocen; no obstante, se cree que hay suficientes recursos para satisfacer la creciente demanda y fortalecer la seguridad energética de muchos países. Sin embargo, la estimulación hidráulica representa uno de los mayores retos de las geociencias ambientales, ya que los impactos sobre el ambiente aún requieren mucha investigación, sobre todo en lo relacionado con modelos numéricos que permitan estimar de manera cuantitativa los impactos y que a su vez hagan posible el diseño de estrategias para mitigar y reducir los impactos de los procesos de exploración y explotación de hidrocarburos en yacimientos no convencionales.

Algunos retos en cuanto a los impactos de la explotación de gas de lutita en el contexto colombiano están asociados también al desarrollo de modelos hidrogeológicos conceptuales que soporten la evaluación del riesgo de contaminación y contribuyan en la reducción de la vulnerabilidad del recurso hídrico. En particular, para ayudar en la construcción de este tipo de modelos se deben instalar pozos piezométricos de control en las zonas cercanas a las explotaciones, que además permitan evaluar los cambios de calidad del agua.

Los recientes estudios [96] reportan que las cantidades hídricas consumidas en el fracking no se comparan con las cantidades consumidas en producción de alimen- 
tos. Ahora bien, se debe considerar los conflictos por uso de agua que se pueden generar en algunas partes del país donde haya proyecciones de explotación de hidrocarburos en yacimientos no convencionales y quw a su vez sean zonas de escases hídrica, por ausencia de fuentes superficiales y bajas precipitaciones.

Adicionalmente, se deben enfocar algunos esfuerzos en el desarrollo de mejores tecnologías de revestimiento en la perforación que las observadas a nivel mundial, para ayudar a mitigar los impactos durante el proceso de perforación. Asimismo, se deben mejorar sustancialmente las actividades preventivas en la gestión de residuos líquidos durante la estimulación hidráulica.

Una tendencia mundial que debe ser tenida en cuenta en el contexto colombiano es la evaluación de otras técnicas para la explotación de gas no convencional que no afecten el recurso hídrico tan significativamente. Por ejemplo, Nielson [26] presentó un procedimiento para evitar la necesidad de fracturar yacimientos, el cual consistía en la gasificación in situ de la lutita mediante un calentador de gas dentro del agujero de perforación. Este procedimiento no generó las mismas tasas de producción que la estimulación hidráulica, así que en ese momento su implementación no fue atractiva para el sector industrial. Pero con el desarrollo de nuevos materiales y nuevos equipos se pueden explorar nuevas tecnologías que permitan reducir los impactos de la estimulación hidráulica en el ambiente.

Resulta fundamental el desarrollo de modelos numéricos durante la fase de solicitud de licencias, principalmente en los lugares donde la vulnerabilidad es mayor. Esto permitiría dimensionar los verdaderos riesgos asociados a la explotación de gas de lutita e incluir en la ecuación los impactos ambientales derivados de esta. Desde esta perspectiva, con el fin de contar con información suficiente para calibrar y validar los modelos, es indispensable exigir la construcción de sistemas robustos de monitoreo de variables de estado y ambientales a los operadores que pretendan realizar actividades de exploración y explotación de gas de lutita.

Como se puede observar en la presente revisión, la explotación de gas de lutita ha hecho importantes contribuciones hacia el mejoramiento de la seguridad energética en diferentes países en los continentes americano y europeo. Sin embargo, en el contexto mundial existen múltiples investigaciones que han sugerido impactos ambientales y sociales relevantes durante los procesos de exploración y explotación de gas de lutita. Esto último impone un reto importante en términos de sostenibilidad global para Colombia, el cual debe garantizar un equilibrio entre los beneficios económicos, el bienestar social y la sostenibilidad ambiental.

\section{AGRADECIMIENTOS}

Los autores agradecen a la Vicerrectoría de Investigación, Ciencia y Tecnología de la Universidad Antonio Nariño por la financiación de la presente investigación en el marco de la convocatoria interna de proyectos de investigación y de jóvenes investigadores. Un agradecimiento especial a los doctores Juan Daniel Valderrama, Edwin González y Andrés 
EFECTOS DE LA ESTIMULACIÓN HIDRÁULICA (FRACKING) EN EL RECURSO HÍDRICO: IMPLICACIONES EN EL CONTEXTO COLOMBIANO

Carvajal por las revisiones realizadas al documento.

\section{REFERENCIAS}

[1] J. Soeder, S. Sharma, N. Pekney et al., "An approach for assessing engineering risk from shale gas wells in the United States," International Journal of Coal Geology, vol. 126, pp. 4-19, 2014. DOI: https://doi.org/10.1016/j. coal.2014.01.004

[2] R. Heinberg, "A brief history of fracking," 2013. [En línea]. Disponible en: http:// www.resilience.org/stories/2013-10-02/ snake-oil-chapter-2-technology-to-therescue/.

[3] R. Heinberg. Snake Oil: How Fracking's False Promise of Plenty Imperils Our Future. Gran Bretaña: Clairwiew Books, 2014.

[4] M. Soliman, J. Daal y L. East, "Fracturing unconventional formations to enhance productivity," Journal of Natural Gas Science and Engineering, vol. 8, pp. 52-67, 2012. DOl: https://doi.org/10.1016/j.jngse.2012.01.007

[5] D. Chuprakov y A. S. Zhubayev, "A variational approach to analyze a natural fault with hydraulic fracture based on the strain energy density criterion," Theoretical and Applied Fracture Mechanics, vol. 53, no. 3, pp. 221-232, 2010. DOI: https://doi.org/10.1016/j. tafmec.2010.06.007

[6] A. Bergmann, F. Weber, H. Meiners et al., "Potential water-related environmental risks of hydraulic fracturing employed in exploration and exploitation of unconventional natural gas reservoirs in Germany," Environmental Sciences Europe, vol. 26, pp.10, 2014. DOl: https:// doi.org/10.1186/2190-4715-26-10

[7] J. Bocora, "Global Prospects for the Development of Unconventional Gas," Procedia - Social and Behavioral Sciences, vol. 65, pp. 436-442, 2012. DOI: https:// doi.org/10.1016/j.sbspro.2012.11.145

[8] M. Kargbo, G. Wilhelm y J. Campbell, "Natural gas plays in the Marcellus shale: Challenges and potential opportunities," Environmental Science \& Technology, vol. 44, no. 15, pp. 5679-5684, 2010. DOI: https://doi.org/10.1021/es903811p

[9] J. Matesanz, "Repercusiones territoriales de la fractura hidráulica o "fracking" en Cantabria, Burgos y Palencia. Los permisos de investigación Bezana y Bigüenzo," 2013. [En línea]. Disponible en: http://eprints.sim.ucm.es/23795/

[10] Revista Semana, "Fracking: una apuesta riesgosa para Colombia," 2016. [En línea]. Disponible en http://sostenibilidad.semana.com/medio-ambiente/ articulo/fracking-una-apuesta-riesgosa-para-colombia/35505

[11] D. Costa, J. Jesus, D. Branco et al., "Extensive review of shale gas environmental impacts from scientific literature (2010-2015)," Environmental Science and Pollution Research, vol. 24, no. 17, pp. 14579-14594, 2017. DOI: https:// doi.org/10.1007/s11356-017-8970-0

[12] G. Speight. The chemistry and technology of petroleum. Boca Ratón: CRC Press, 2014. 
[13] Ecopetrol, "Qué es el gas natural," 2014. [En línea]. Disponible en http:// www.ecopetrol.com.co/wps/portal/ es/ecopetrol-web/productos-yservicios/productos/gas-natural/ Informaci\%C3\%B3n\%20General/quees-el-gas-natural/!ut/p/z0/04_Sj9CPyk ssy0xPLMnMz0vMAfljo8ziLQIMHd09 DQy9DZwt3QwcjTwsQxw9g4I8nlz0C7 IdFQEONbdQ/

[14] T. Eaton, "Science-based decision-making on complex issues: Marcellus shale gas hydrofracking and New York City water supply," Science of The Total Environment, vols. 461-462, pp. 158-169, 2013. DOI: https://doi.org/10.1016/j. scitotenv.2013.04.093

[15] S. G. Osborn, A. Vengosh, N. R. Warner et al., "Methane contamination of drinking water accompanying gaswell drilling and hydraulic fracturing," Proceedings of the National Academy of Sciences, vol. 108, no. 20, pp. 8172-8176, 2011. DOI: https://doi. org/10.1073/pnas. 1100682108

[16] C. G. Groat y T. W. Grimshaw. Factbased regulation for environmental protection in shale gas development. Austin: The Energy Institute, University of Texas at Austin, 2012.

[17] N. Maricic, S. D. Mohaghegh y E. Artun, "A parametric study on the benefits of drilling horizontal and multilateral wells in coalbed methane reservoirs," SPE Reservoir Evaluation \& Engineering, vol. 11, no. 06, pp. 976-983, 2008. DOI: https://doi.org/10.2118/96018-PA
[18] J. Caputo, "Shale plays: basic geological and engineering concepts. American Association of Profesional Landmen," 2011. [En línea]. Disponible en: http://www.landman.org/docs/whitepapers/03-caputo_aapl_shale_play_ seminar_part1_final.pdf

[19] S. J. P. Oliver, V. A. Kuuskraa, C. F. Brandenburg et al., "The Antrim Shale of the Michigan Basin: An Active Devonian Shale Gas Play," en SPE Gas Technology Symp., pp. 487-494, 1989. DOI: https://doi.org/10.2118/19104-ms

[20] J. D. Arthur, B. K. Bohm, B. J. Coughlin et al., "Evaluating the environmental implications of hydraulic fracturing in shale gas reservoirs," en SPE Americas E\&P Environmental and Safety Conf., pp. 1-15, 2009. DOI: https://doi. org/10.2118/121038-ms

[21] A. Ybáñez, A., "Nociones de ingeniería aplicada a reservorios no convencionales," Petrotecnia, pp. 1-9, 2009.

[22] J. D. Arthur, B. Bohm y M. Layne, "Hydraulic fracturing considerations for natural gas wells of the Marcellus Shale," The Gulf Coast Association of Geological Societies Transactions, vol. 59, pp. 49-59, 2009.

[23] K. Williams, "The Permeability of Overpressure Shale Seals and of Source Rock Reservoirs is the Same," Search and Discovery, art. 40935, pp. 1-10, 2012.

[24] T. Myers, "Potential contaminant pathways from hydraulically fractured shale to aquifers," Groundwater, vol. 50 no. 6, pp. 872-882, 2012. 
EFECTOS DE LA ESTIMULACIÓN HIDRÁULICA (FRACKING) EN EL RECURSO HÍDRICO: IMPLICACIONES EN EL CONTEXTO COLOMBIANO

DOI: https://doi.org/10.1111/j.17456584.2012.00933.x

[25] S. Ghoshray, "Powering the future: a 21st century guide for energy practitioners: charting the future trajectory for fracking regulation: from environmental democracy to cooperative federalism," Thurgood Marshall Law Review, vol. 38, pp. 199-321, 2013.

[26] D. H. Nielson, "Method and apparatus for shale gas recovery. Google Patents," 1990. [En línea]. Disponible en: https://www.google.com/patents/ US4928765

[27] L. M. McKenzie, R. Z. Witter, L. S. Newman et al., "Human health risk assessment of air emissions from development of unconventional natural gas resources," Science of the Total Environment, vol. 424, pp. 79-87, 2012. DOI: https://doi.org/10.1016/j. scitotenv.2012.02.018

[28] U.S. Energy Information Administration (EIA), "World Shale Gas". [En línea]. Disponible en: https://www.eia. gov/analysis/studies/worldshalegas/

[29] W. Ding, D. Zhu, J. Cai et al., "Analysis of the developmental characteristics and major regulating factors of fractures in marine-continental transitional shalegas reservoirs: A case study of the Carboniferous-Permian strata in the southeastern Ordos Basin, central China," Marine and Petroleum Geology, vol. 45, pp. 121-133, 2013. DOI: https://doi. org/10.1016/j.marpetgeo.2013.04.022

[30] C. Jia, M. Zheng y Y. Zhang, Y., "Unconventional hydrocarbon resources in China and the prospect of exploration and development," Petroleum Exploration and Development, vol. 39, no. 2, pp. 139-146, 2012. DOI: https://doi. org/10.1016/S1876-3804(12)60026-3

[31] A. D. Decker, D. G. Hill y D. E. Wicks, D. E., "Log-Based Gas Content and Resource Estimates for the Antrim Shale Michigan Basin," Society of Petroleum Engineers, pp. 659-669, 1993. DOI: https:// doi.org/10.2118/25910-MS

[32] L. Galvan, C. Guédez y R. Reyes, "Los macroprocesos de la industria petrolera y sus consecuencias ambientales," Universidad, ciencia y tecnología, vol. 11, no. 43, pp. 91-97, 2007.

[33] M. C. Mei-in y D. R. Dickerson, "Pyrolysis of Eastern Gas Shales--Effects of Temperature and Atmosphere: ABSTRACT". AAPG Bulletin, vol. 63, no. 9, pp.1577-1577, 1979.

[34] A. M. Romero y R. P. Philp, "Organic geochemistry of the Woodford Shale, southeastern Oklahoma: How variable can shales be?," AAPG Bulletin, vol. 96, no. 3, pp. 493-517, 2012. DOI: https:// doi.org/10.1306/08101110194

[35] S. Jenner y A. J. Lamadrid, "Shale gas vs. coal: Policy implications from environmental impact comparisons of shale gas, conventional gas, and coal on air, water, and land in the United States," Energy Policy, vol. 53, pp. 442-453, 2013. DOI: https://doi.org/10.1016/j. enpol.2012.11.010

[36] J. G. Henry y G. W. Heinke. Ingeniería ambiental. México, D. F.: Pearson Educación, 1999. 
[37] R. E. Bishop, D. J. Lampe, B. W. Okey et al., "Fracking: A Roundtable," Journal of Appalachian Studies, vol. 18, no. 1-2, pp. 31-47, 2012.

[38] P. Peduzzi y R. Harding, "Gas fracking: ¿can we safely squeeze the rocks?," Environmental Development, vol. 6, pp. 86-99, 2013. DOI: https://doi.org/10.1016/j.envdev.2012.12.001

[39] R. Weijermars, G. Drijkoningen, T. J. Heimovaara et al., "Unconventional gas research initiative for clean energy transition in Europe," Journal of Natural Gas Science and Engineering, vol. 3, no. 2, pp. 402-412, 2011. DOI: https://doi. org/10.1016/j.jngse.2011.04.002

[40] M. Guarnone, F. Rossi, E. Negri et al., "An unconventional mindset for shale gas surface facilities," Journal of Natural Gas Science and Engineering, vol. 6, pp.14-23, 2012. DOI: https:// doi.org/10.1016/j.jngse.2012.01.002

[41] A. Blohm, J. Peichel, C. Smith et al., "The significance of regulation and land use patterns on natural gas resource estimates in the Marcellus shale," Energy Policy, vol. 50, pp. 358-369, 2012. DOI: https://doi.org/10.1016/j. enpol.2012.07.031

[42] S. L. Brantley, D. Yoxtheimer, S. Arjmand et al., "Water Resource Impacts during Unconventional Shale Gas Development: the Pennsylvania Experience," International Journal of Coal Geology, vol. 126, pp. 140-156, 2014. DOI: https://doi.org/10.1016/j. coal.2013.12.017
[43] R. D. Vidic, S. L. Brantley, J. Vandenbossche et al., "Impact of Shale Gas Development on Regional Water Quality," Science, vol. 340, pp. 1-11, 2013. DOl: https:// doi.org/10.1126/science.1235009

[44] J. Kriesky, B. D. Goldstein, K. Zell y S. Beach, "Differing opinions about natural gas drilling in two adjacent counties with different levels of drilling activity," Energy Policy, vol. 58, pp. 228-236, 2013. DOI: https://doi.org/10.1016/j. enpol.2013.03.005

[45] L. O. Haluszczak, A. W. Rose y L. R. Kump, "Geochemical evaluation of flowback brine from Marcellus gas wells in Pennsylvania, USA," Applied Geochemistry, vol. 28, pp. 55-61, 2013. DOI: https://doi.org/10.1016/j.apgeochem.2012.10.002

[46] S. Lechtenböhmer, M. Atmann, S. Capito et al., "Repercusiones de la extracción de gas y petróleo de esquisto en el medio ambiente y la salud humana," Bruselas: Departamento Temático de Política Económica y Científica, Parlamento Europeo, 2011. [En línea]. Disponible en: http://www.fracturahidraulicano.info/sites/default/files/media/ documentos/shale-gas_parlamento_ europeo-es_0.pdf

[47] S. Kovats, M. Depledge, A. Haines et al., "The health implications of fracking," The Lancet, vol. 383, no. 9919, pp. 757-758, 2014. DOI: https://doi. org/10.1016/S0140-6736(13)62700-2

[48] D. D. Schafer, "Frack Attack: Cracking the Case against Hydraulic Fracturing," 
2012. [En línea]. Disponible en: https:// i2i.org/wp-content/uploads/2012/07/ IP_10_2012_c.pdf

[49] G. L. Miller y H. K. Barthel, "Drilling mud-cement compositions for well cementing operations," U.S. Patent no. 3887009, Jun. 6, 1975.

[50] H. C. Darley y G. R. Gray. Composition and properties of drilling and completion fluids. Houston: Gulf Professional Publishing, 1998.

[51] E. Post y W. Oldham, "Composition for drilling muds," U.S. Patent no 2579453, Dic. 25, 1951.

[52] Asamblea contra la Fractura Hidráulica, "La extracción de gas no convencional y la fractura hidráulica permisos en Burgos," pp. 1-24, 2011. [En línea]. Disponible: https://proyectourraca.files.wordpress.com/2012/02/ informe-asamblea-burgos-2011.pdf

[53] M. Ratner y M. Tiemann, "An overview of unconventional oil and natural gas: Resources and federal actions," Congressional Research Service, 2015. [En línea]. Disponible en: https://fas.org/ sgp/crs/misc/R43148.pdf

[54] N. R. Warner, R. B. Jackson, T. H. Darrah et al., "Geochemical evidence for possible natural migration of Marcellus Formation brine to shallow aquifers in Pennsylvania," Proceedings of the National Academy of Sciences, vol. 109, no. 30, pp. 11961-11966, 2012. DOI: https:// doi.org/10.1073/pnas.1121181109

[55] R. W. Howarth, R. Santoro y A. Ingraffea, "Methane and the greenhouse-gas foo- tprint of natural gas from shale formations," Climatic Change, vol. 106, no. 4, pp. 679-690, 2011. DOI: https://doi. org/10.1007/s10584-011-0061-5

[56] A. Vengosh, N. Warner, R. Jackson y T. Darrah, "The effects of shale gas exploration and hydraulic fracturing on the quality of water resources in the United States," Procedia Earth and Planetary Science, vol. 7, pp. 863-866, 2013. DOI: https://doi.org/10.1016/j. proeps.2013.03.213

[57] A. Vengosh, R. B. Jackson, N. Warner et al., "A Critical Review of the Risks to Water Resources from Unconventional Shale Gas Development and Hydraulic Fracturing in the United States," Environmental Science \& Technology, vol. 48 , no. 15 , pp. $8334-8348,2014$. DOI: https://doi.org/10.1021/es405118y

[58] J. Hoffman, "Potential Health and Environmental Effects of Hydrofracking in the Williston Basin, Montana," 2012. [En línea]. Disponible en: http:// serc.carleton.edu/NAGTWorkshops/ health/case_studies/hydrofracking_w.html

[59] R. B. Jackson, A. Vengosh, J. W. Carey et al., "The environmental costs and benefits of fracking," Annual Review of Environment and Resources, vol. 39, pp. 327362, 2014. DOI: https://doi.org/10.1146/ annurev-environ-031113-144051

[60] D. J. Soeder y W. M. Kappel. Water resources and natural gas production from the Marcellus Shale. Reston, Virginia: US Department of the Interior, US Geological Survey, 2009. 
[61] X. E. Refunjol, K. J. Marfurt y J. H. Le Calvez, "Inversion and attribute-assisted hydraulically induced microseismic fracture characterization in the North Texas Barnett Shale," The Leading Edge, vol. 30, no. 3, pp. 292-299, 2011. DOI: https://doi.org/10.1190/1.3567260

[62] N. R. Warner, T. M. Kresse, P. D. Hays et al., "Geochemical and isotopic variations in shallow groundwater in areas of the Fayetteville shale development, north-central Arkansas," Applied Geochemistry, vol. 35, pp. 207-220, 2013. DOI: https://doi.org/10.1016/j.apgeochem.2013.04.013

[63] J. L. Northrup, "The Unique Environmental Impacts of Horizontally Hydrofracking Shale," Otsego 2000, pp. 2-5, 2010. [En línea]. Disponible en: http:// www.otsego2000.org/documents/ 10aug03_Northrup_EPA_final.pdf

[64] J. F. Gale, R. M. Reed y J. Holder, "Natural fractures in the Barnett Shale and their importance for hydraulic fracture treatments," AAPG Bulletin, vol. 91, no. 4, pp. 603-622. DOI: https://doi. org/10.1306/11010606061

[65] J. R. Plukas, "Energy Law-Not Balancing Well Interests Well: How the Supreme Court of Pennsylvania Mismanaged Oil and Gas Lease Policy in TW Philips Gas \& Oil Co. v. Jedlicka," New Eng. L. Rev., vol. 36, pp. 68-68, 2014.

[66] D. Rahm, "Regulating hydraulic fracturing in shale gas plays: The case of Texas," Energy Policy, vol. 39, no. 5, pp. 2974-2981, 2011. DOI: https://doi. org/10.1016/j.enpol.2011.03.009
[67] Q. Wang, X. Chen, A. N. Jha et al., "Natural gas from shale formation-The evolution, evidences and challenges of shale gas revolution in United States," Renewable and Sustainable Energy Reviews, vol. 30, pp. 1-28, 2014. DOI: https://doi.org/10.1016/j.rser.2013.08.065

[68] Q. Jiang, J. Rentschler, R. Perrone y K. Liu "Application of ceramic membrane and ion-exchange for the treatment of the flowback water from Marcellus shale gas production," Journal of Membrane Science, vol. 431, pp. 55-61, 2013. DOI: https://doi.org/10.1016/j. memsci.2012.12.030

[69] L. S. Lauver, "Environmental health advocacy: an overview of natural gas drilling in northeast Pennsylvania and implications for pediatric nursing," Journal of Pediatric Nursing, vol. 27, no. 4, pp. 383-389, 2012. DOI: https:// doi.org/10.1016/j.pedn.2011.07.012

[70] W. Orem, C. Tatu, M. Varonka et al., "Organic substances in produced and formation water from unconventional natural gas extraction in coal and shale," International Journal of Coal Geology, vol. 126, pp. 20-31, 2014. DOI: https:// doi.org/10.1016/j.coal.2014.01.003

[71] M. A. Engle y E. L. Rowan, "Geochemical evolution of produced waters from hydraulic fracturing of the Marcellus Shale, northern Appalachian Basin: A multivariate compositional data analysis approach," International Journal of Coal Geology, vol. 126, pp. 45-56, 2013. DOI: https://doi.org/10.1016/j. coal.2013.11.010 
[72] A. G. Bunch, C. S. Perry, L. Abraham et al., "Evaluation of impact of shale gas operations in the Barnett Shale region on volatile organic compounds in air and potential human health risks," Science of the Total Environment, vol. 468, pp. 832-842, 2014. DOI: https://doi.org/10.1016/j. scitotenv.2013.08.080

[73] N. G. Phillips, R. Ackley, E. R. Crosson et al., "Mapping urban pipeline leaks: Methane leaks across Boston," Environmental pollution, vol. 173, pp. 1-4, 2013. DOI: https://doi.org/10.1016/j. envpol.2012.11.003

[74] J. Fox. Gasland, "International Wow Company," 2011. [En línea]. Disponible en: http://www.hbo.com/documentaries/gasland

[75] M. Bamberger y R. E. Oswald, "Impacts of gas drilling on human and animal health," Journal of Environmental and Occupational Health Policy, vol. 22, no. 1, pp. 51-77, 2012. DOI: https://doi. org/10.2190/NS.22.1.e

[76] D. J. Soeder, "Porosity and permeability of eastern Devonian gas shale," Society of Petroleum Engineers, vol. 3, no. 01, pp. 116-124, 1998. DOI: https://doi. org/10.2118/15213-PA

[77] C. Rivard, D. Lavoie, R. Lefebvre et al., "An overview of Canadian shale gas production and environmental concerns," International Journal of Coal Geology, vol. 126, pp. 64-76, 2013. DOI: https:// doi.org/10.1016/j.coal.2013.12.004

[78] Servicio Geológico de Estados Unidos (USGS), "Water Use in the United Sta- tes," 2014. [En línea]. Disponible en: http://water.usgs.gov/watuse/

[79] J. P. Nicot y B. R. Scanlon, "Water use for shale-gas production in Texas, U.S.," Environmental science and technology, vol. 46, no. 6, pp. 3580-3586, 2012. DOI: https://doi.org/10.1021/ es204602t

[80] M. Blauch, N. Houston, M. Seyman et al., "Technique Reuses Water in Shale," American Oil \& Gas Reporter, 2009.

[81] C. R. Clarkson, "Production data analysis of unconventional gas wells: Review of theory and best practices," International Journal of Coal Geology, vols. 109-110, pp. 101-146, 2013. DOI: https://doi.org/10.1016/j. coal.2013.01.002

[82] Servicio Geológico de Estados Unidos (USGS), "New Insight on Ground Shaking from Man-Made Earthquakes," 2015. [En línea]. Disponible en: https://www.usgs.gov/news/newinsight-ground-shaking-man-madeearthquakes

[83] M. D. Petersen, C. S. Mueller, M. P. Moschetti et al. "2016 one-year seismic hazard forecast for the Central and Eastern United States from induced and natural earthquakes," Reston: US Geological Survey, 2016. DOI: https://doi. org/10.3133/ofr20161035

[84] B. A. Watson, "Fracking and Cracking: Strict Liability for Earthquake Damage Due to Wastewater Injection and Hydraulic Fracturing," 2016. [En línea]. Disponible en: https://papers.ssrn.com/ sol3/papers.cfm?abstract_id=2735862 
[85] C. Schultz, "Marcellus Shale fracking waste caused earthquakes in Ohio," Eos, Transactions American Geophysical Union, vol. 94, no. 33, pp. 296-296, 2013. DOI: https://doi.org/10.1002/2013E0330008

[86] K. C. Wilson y L. J. Durlofsky, "Optimization of shale gas field development using direct search techniques and reduced-physics models," Journal of Petroleum Science and Engineering, vol. 108, pp. 304-315, 2013. DOI: https:// doi.org/10.1016/j.petrol.2013.04.019

[87] Z. Caineng, Z. Guangya, T. Shizhen et al., "Geological features, major discoveries and unconventional petroleum geology in the global petroleum exploration," Petroleum Exploration and Development, vol. 37, no. 2, pp. 129-145, 2010. DOI: https://doi.org/10.1016/ S1876-3804(10)60021-3

[88] W. Sang, C. R. Stoof, W. Zhang et al., "Effect of Hydrofracking fluid on colloid transport in the unsaturated zone," Environmental Science and Technology, vol. 48, no. 14, pp. 8266-8274, 2014. DOI: https://doi.org/10.1021/ es501441e

[89] J. Rutqvist, A. P. Rinaldi, E. Cappa y G. J. Mordis, "Modeling of fault reactivation and induced seismicity during hydraulic fracturing of shale-gas reservoirs," Journal of Petroleum Science and Engineering, vol. 107, pp. 31-44, 2013. DOI: https://doi.org/10.1016/j. petrol.2013.04.023

[90] C. Gassiat, T. Gleeson, R. Lefebvre y J. McKenzie "Hydraulic fracturing in faulted sedimentary basins: Numerical simulation of potential contamination of shallow aquifers over long time scales: hydraulic fracturing in faulted sedimentary basins," Water Resources Research, vol. 49, no. 12, pp. 8310-8327, 2013. DOI: https://doi.org/10.1002/2013WR014287

[91] L. D. Donado, J. C. Buitrago, M. C. Vargas et al., "Evaluación de las condiciones de uso del agua subterránea en la zona de recarga del acuífero Morroa, Colombia," 2002. [En línea] Disponible en: http://www.docentes.unal.edu.co/ Iddonadog/docs/Presentations/Donado_2002d.pdf

[92] M. Zoback, S. Kitasei y B. Copithorne. Addressing the environmental risks from shale gas development. Washington, D.C.: Worldwatch Institute, 2010.

[93] J. Huang, Z. O. U. Caineng, L. I. Jianzhong et al., "Shale gas generation and potential of the lower Cambrian Qiongzhusi formation in the southern Sichuan Basin, China," Petroleum Exploration and Development, vol. 39, no. 1, pp. 75-81, 2012. DOI: https://doi. org/10.1016/S1876-3804(12)60017-2

[94] O. Navas. "Anatomía geológica de Colombia," Sociedad Colombiana de Geología, 2003.

[95] S. Noguera, "El costo humano del petróleo en Puerto Gaitán," 2016. [En línea]. Disponible en: http://www. elespectador.com/noticias/nacional/ el-costo-humano-del-petroleo-puerto-gaitan-articulo-644426 
[96] B. R. Scanlon, B. L. Ruddell, P. M. Reed et al., "The food-energy-water nexus: Transforming science for so- ciety," Water Resources Research, pp. 1-12, 2017. DOI: https://doi.or$\mathrm{g} / 10.1002 / 2017 \mathrm{wr} 020889$ 